\title{
Nonexistence Results for Higher Order Fractional Differential Inequalities with Nonlinearities Involving Caputo Fractional Derivative
}

\author{
Mohamed Jleli $^{1}$ (D), Bessem Samet ${ }^{1}$ (D) and Calogero Vetro ${ }^{2, *(D)}$ \\ 1 Department of Mathematics, College of Science, King Saud University, P.O. Box 2455, \\ Riyadh 11451, Saudi Arabia; jleli@ksu.edu.sa (M.J.); bsamet@ksu.edu.sa (B.S.) \\ 2 Department of Mathematics and Computer Science, University of Palermo, Via Archirafi 34, \\ 90123 Palermo, Italy \\ * Correspondence: calogero.vetro@unipa.it
}

Citation: Jleli, M.; Samet, B.; Vetro, C. Nonexistence Results for Higher Order Fractional Differential Inequalities with Nonlinearities Involving Caputo Fractional Derivative. Mathematics 2021, 9, 1866. https://doi.org/10.3390/ math9161866

Academic Editor: Ivan Matychyn

Received: 28 June 2021

Accepted: 3 August 2021

Published: 6 August 2021

Publisher's Note: MDPI stays neutral with regard to jurisdictional claims in published maps and institutional affiliations.

Copyright: (c) 2021 by the authors. Licensee MDPI, Basel, Switzerland. This article is an open access article distributed under the terms and conditions of the Creative Commons Attribution (CC BY) license (https:// creativecommons.org/licenses/by/ $4.0 /)$.

\begin{abstract}
Higher order fractional differential equations are important tools to deal with precise models of materials with hereditary and memory effects. Moreover, fractional differential inequalities are useful to establish the properties of solutions of different problems in biomathematics and flow phenomena. In the present work, we are concerned with the nonexistence of global solutions to a higher order fractional differential inequality with a nonlinearity involving Caputo fractional derivative. Namely, using nonlinear capacity estimates, we obtain sufficient conditions for which we have no global solutions. The a priori estimates of the structure of solutions are obtained by a precise analysis of the integral form of the inequality with appropriate choice of test function.
\end{abstract}

Keywords: higher order fractional differential inequality; global solution; nonexistence; test function method; reaction-diffusion process

\section{Introduction}

Differential equations of fractional order are a interesting model in different areas of engineering sciences such as modeling of materials with memory and hereditary effects (see, for example, the books [1,2]). Additionally, the study of fractional differential inequalities leads to establish the properties of solutions to electrodynamic and viscosity systems (for applications to diffusion processes see [3]). Therefore, the investigation of global solutions to different classes of fractional differential equations and inequalities has been a point of interest for many researchers. This study requires the knowledge of mathematical methods and their impact in applied sciences, without forgetting their contribution to fundamental research in topological spaces and geometric conditions. Indeed, despite many advances in the field, understanding the dynamic behavior associated to different systems still gives mathematical difficulties and opens significant challenges. With regard to the existence of global solutions, many results have been reported in the literature (see, e.g., [4-7] and the references therein). In [4], the authors apply continuation methods for contractive maps to fractional differential equations. In [5], useful properties of Mittag-Leffler functions, together with fixed-point methods, lead to the existence and uniqueness of global solutions in the case of delay fractional differential equations. Concerning the nonexistence of global solutions, we mention the papers [8-14] and the references therein. For instance, ref. [9] shows that fractional derivatives of lower order have a strong influence on the character of the solutions. Reference [14] concerns both the existence and nonexistence of positive solutions for a parametric problem when the reaction term presents superlinear and sublinear growths, as the parameter lies in certain intervals. This kind of results helps in developing control of system shapes (see, e.g., [15]), but also in numerical approximation of solutions in diffusion processes (see, e.g., [16]). 
In the present work, we study the problem

$$
\left\{\begin{array}{l}
{ }^{C} D_{0}^{1+\alpha} y(t)+{ }^{C} D_{0}^{1+\beta} y(t) \geq\left|{ }^{C} D_{0}^{\gamma} y(t)\right|^{q}+\vartheta(t), \quad t>0, \\
y(0)=y_{0}, y^{\prime}(0)=y_{1} \ldots, y^{(n-1)}(0)=y_{n-1},
\end{array}\right.
$$

where $q>1, n \geq 2$ is a natural number, $y_{0} \in \mathbb{R}, y_{i} \geq 0, i=1,2, \ldots, n-1, \vartheta \in C([0, \infty))$, $\vartheta \geq 0, \alpha, \beta \in(n-2, n-1), \gamma \in(0,1)$, and ${ }^{C} D_{0}^{r}$, with $r \in\{1+\alpha, 1+\beta, \gamma\}$, is the Caputo fractional derivative of order $r$. Our aim is to derive criteria for which the considered problem admits no global solution (i.e., we focus on the nonexistence problem). The key ingredient in the sequel, is the nonlinear capacity method introduced by Mitidieri and Pohozaev (see [17]). This approach works well for higher order operators that fail to satisfy any comparison principles. The main idea is to establish certain (sufficient) conditions of non-solvability of inequalities on functional classes (here we deal with absolutely continuous functions; $A C$ for short). The method develops a priori estimates of the structure of solutions from a precise analysis of certain integrals related to the involved inequality. To apply the method, each special differential problem requires a particular choice of test functions and related geometrical structure of their support.

We mention the possibility of short-memory application of (1), referring the readers to the works [18] (model of abdominal aortic aneurysm phenomena), [19] (Noether's theorems of fractional generalized Birkhoffian systems in terms of classical and combined Caputo derivatives), and the comprehensive book [20]. Therefore, as potential real-life applications of Equation (1), we bring (new) epidemiological systems via Caputo fractional order operator which is both a nonlocal operator and possesses features related to memory of the epidemic (see, for example [21]). Moreover, we know that the Laplace transform of the Caputo fractional derivative can be seen as a generalization of Laplace transform of integer order derivative, changing $n$ by $r$. This feature is not true in the case of RiemannLiouville fractional derivative. In general, the results herein strongly depend on the used fractional operator (that is, the Caputo fractional derivative).

Next, we briefly recall some results from the literature which are related to our work here. In [10], the authors considered the problem

$$
\left\{\begin{array}{l}
{ }^{C} D_{0}^{1+\alpha} y(t)+{ }^{C} D_{0}^{1+\beta} y(t) \geq\left|{ }^{C} D_{0}^{\gamma} y(t)\right|^{q}, \quad t>0, \\
y(0)=y_{0}, y^{\prime}(0)=y_{1},
\end{array}\right.
$$

where $q>1, \alpha, \beta, \gamma \in(0,1), y_{0} \in \mathbb{R}$, and $y_{1} \geq 0$. Notice that problem (2) is a special case of problem (1) with $n=2$ and $\vartheta \equiv 0$. Namely, ref. [10] discussed the cases $y_{1}>0$ and $y_{1}=0$. In the case where $y_{1}>0$, the authors proved that for all $q>1$, problem (2) admits no global solution. In the case of $y_{1}=0$, the authors established that, if $\gamma \leq \min \{\alpha, \beta\}$, then for all $q>1$, the only global solution to problem (2) is $y \equiv y_{0}$, while, if $\gamma>\min \{\alpha, \beta\}$, then for all $1<q<\frac{1}{\gamma-\min \{\alpha, \beta\}}$, the only global solution to problem (2) is $y \equiv y_{0}$.

Summarizing, we extend the obtained results in [10] to higher order fractional differential inequalities of type (1). We first consider the case $\vartheta \equiv 0$. Next, we study the case where $\vartheta \not \equiv 0$ (the inhomogeneous case). Therefore, this is a new step forward in providing additional knowledge to the study of qualitative behavior of solutions. Indeed, it is widely recognized that the mathematical features of solutions provide a fundamental starting point to the physical understanding of any diffusion processes.

Before presenting our main results, let us mention what we mean by a global solution to problem (1).

Definition 1. A global solution to problem (1) is a function $y \in A C^{n}([0, \infty))$ satisfying

$$
{ }^{C} D_{0}^{1+\alpha} y(t)+{ }^{C} D_{0}^{1+\beta} y(t) \geq\left|{ }^{C} D_{0}^{\gamma} y(t)\right|^{q}+\vartheta(t),
$$


for almost everywhere $t \geq 0$, and the initial conditions

$$
y(0)=y_{0}, y^{\prime}(0)=y_{1} \ldots, y^{(n-1)}(0)=y_{n-1} .
$$

Consider now problem (1) with $\vartheta \equiv 0$. To make reading easier, we first discuss the case where $y_{j}>0$ for some $j \in\{1,2, \ldots, n-1\}$.

Theorem 1. Let $\vartheta \equiv 0, n \geq 2, \alpha, \beta \in(n-2, n-1), \gamma \in(0,1), y_{0} \in \mathbb{R}$, and $y_{i} \geq 0$ for all $i=1,2, \ldots, n-1$. Suppose that there exists $j \in\{1,2, \ldots, n-1\}$, such that $y_{j}>0$. Then, for all $q>1$, problem (1) admits no global solution.

Next, we go deep into the case where $y_{i}=0$, for all $i \in\{1,2, \ldots, n-1\}$.

Theorem 2. Let $\vartheta \equiv 0, n \geq 2, n-2<\alpha \leq \beta<n-1, \gamma \in(0,1)$, and $y_{0} \in \mathbb{R}$. Suppose that

$$
y_{i}=0, \quad i=1,2, \ldots, n-1 .
$$

(i) If $\gamma \leq \alpha$, then, for all $q>1$, the only global solution to problem (1) is $y \equiv y_{0}$.

(ii) If $\gamma>\alpha$, then, for all

$$
1<q<\frac{1}{\gamma-\alpha}
$$

the only global solution to problem (1) is $y \equiv y_{0}$.

For the inhomogeneous case, we have the following result.

Theorem 3. Let $\vartheta \in C([0, \infty)), \vartheta \geq 0, n \geq 2, n-2<\alpha \leq \beta<n-1, \gamma \in(0,1)$, and $y_{0} \in \mathbb{R}$. Suppose that (3) is satisfied.

(i) If $\gamma \leq \alpha$, then, for all $q>1$, problem (1) has no global solution.

(ii) If $\gamma>\alpha$ and

$$
\limsup _{T \rightarrow \infty} T^{-\frac{(\gamma-\alpha-1) q}{q-1}-1} \int_{0}^{T} \vartheta(t) d t=\infty,
$$

then problem (1) has no global solution.

Consider the special case where

$$
\vartheta(t)=(t+1)^{\rho}, \quad t \geq 0,
$$

where $\rho>-1$. From Theorem 3 , we deduce the following result.

Corollary 1. Let $\vartheta$ be the function given by (5), $n \geq 2, n-2<\alpha \leq \beta<n-1, \gamma \in(0,1)$, and $y_{0} \in \mathbb{R}$. Suppose that (3) is satisfied. If $\gamma>\alpha$ and $\gamma-\alpha-1<\rho \leq 0$, then for all $q>1$, problem (1) has no global solution.

As mentioned above, the proofs of Theorems 1-3 are based on the nonlinear capacity method (see [17]) and some useful integral estimates. Therefore, we use general rules, but their applications strongly depend on the precise form of the investigated nonlinearity.

The rest of the paper is organized as follows. In Section 2, we recall some basic notions on fractional calculus, and establish preliminary results that will be used in the proofs of the main theorems. In Section 3, we give the detailed proofs of Theorems 1-3.

\section{Preliminaries}

Let $A C\left(\mathbb{R}_{+}\right)$be the space of absolutely continuous functions on the positive semiaxis $\mathbb{R}_{+}=[0, \infty)$. Given a natural number $n \geq 2$, we denote by $A C^{n}\left(\mathbb{R}_{+}\right)$the space of functions which have continuous derivatives up to order $n-1$ on $\mathbb{R}_{+}$such that $f^{(n-1)} \in A C\left(\mathbb{R}_{+}\right)$. Here, for a positive natural number $i, f^{(i)}$ denotes the derivative of order $i$ of $f$. Different 
from the derivative of integer order, there are several definitions for derivatives of fractional order. We point out that these notions are generally not equivalent each other. Therefore, we recall the definitions related to our finding here.

Let $0<T<\infty$ be fixed. Given $f \in L^{1}(0, T)$, the left-sided Riemann-Liouville fractional integral of order $\sigma>0$ of $f$ is given by

$$
I_{0}^{\sigma} f(t)=\frac{1}{\Gamma(\sigma)} \int_{0}^{t}(t-s)^{\sigma-1} f(s) d s,
$$

for almost everywhere $t \in[0, T]$. The right-sided Riemann-Liouville fractional integral of order $\sigma>0$ of $f$ is given by

$$
I_{T}^{\sigma} f(t)=\frac{1}{\Gamma(\sigma)} \int_{t}^{T}(s-t)^{\sigma-1} f(s) d s,
$$

for almost everywhere $t \in[0, T]$. Here, $\Gamma(\cdot)$ is the Gamma function (see, for example [3], p. 24).

By combining the above notions, we recall some properties in the following lemmas.

Lemma 1 (see [1]). Let $\sigma, \delta>0$ and $f \in L^{\kappa}(0, T)$, where $1 \leq \kappa \leq \infty$. Then

$$
I_{0}^{\sigma}\left(I_{0}^{\delta} f\right)(t)=I_{0}^{\delta}\left(I_{0}^{\sigma} f\right)(t)=I_{0}^{\sigma+\delta} f(t),
$$

for almost everywhere $t \in[0, T]$.

Lemma 2 (see [1]). Let $\rho>0, \tau, \mu \geq 1$, and $\frac{1}{\tau}+\frac{1}{\mu} \leq 1+\rho(\tau \neq 1, \mu \neq 1$, in the case $\left.\frac{1}{\tau}+\frac{1}{\mu}=1+\rho\right)$. If $(f, g) \in L^{\tau}(0, T) \times L^{\mu}(0, T)$, then

$$
\int_{0}^{T} I_{0}^{\rho} f(t) g(t) d t=\int_{0}^{T} f(t) I_{T}^{\rho} g(t) d t .
$$

Given $\sigma \in(n-1, n)$, where $n \geq 1$ is a natural number, the (left-sided) Caputo fractional derivative of order $\sigma$ of $f \in A C^{n}\left(\mathbb{R}_{+}\right)$is defined by

$$
{ }^{C} D_{0}^{\sigma} f(t)=I_{0}^{n-\sigma} f^{(n)}(t),
$$

for almost everywhere $t \in[0, T]$. For more details about the above concepts, see also $[2,3,20]$.

As mentioned in Section 1, Mitidieri and Pohozaev showed how appropriate choices of the test function lead to nonexistence results to differential problems (see again [17]). Basically, the test functions are infinitely differentiable functions with compact support. Here, to implement a similar strategy as the one in [17], we define the test function

$$
\varphi(t)=T^{-\lambda}(T-t)^{\lambda}, \quad 0 \leq t \leq T, \quad \text { for } \lambda \gg 1 \text { (i.e., } \lambda \text { is large enough) }
$$

We establish the following auxiliary lemma for $\varphi(\cdot)$.

Lemma 3. Let $n \geq 2$ be a natural number, $r \in(n-2, n-1)$ and $\delta>0$. Then

$$
\begin{aligned}
I_{T}^{n-1-r} \varphi(t) & =\frac{\Gamma(\lambda+1)}{\Gamma(n+\lambda-r)} T^{-\lambda}(T-t)^{n-1-r+\lambda}, \\
\left(I_{T}^{n-1-r} \varphi\right)^{(i)}(t) & =\frac{(-1)^{i} \Gamma(\lambda+1)}{\Gamma(n+\lambda-r-i)} T^{-\lambda}(T-t)^{n-i-1-r+\lambda}, i=1, \ldots, n, \\
I_{T}^{\delta}\left[\left(I_{T}^{n-1-r} \varphi\right)^{(n)}\right](t) & =\frac{(-1)^{n} \Gamma(\lambda+1)}{\Gamma(\delta+\lambda-r)} T^{-\lambda}(T-t)^{\delta+\lambda-r-1} .
\end{aligned}
$$


Proof. By considering the right-sided Riemann-Liouville fractional integral of order $n-1-r$ (i.e., $\sigma:=n-1-r>0$ in (6)) of $\varphi(\cdot)$ (given by (7)), we have

$$
\begin{aligned}
I_{T}^{n-1-r} \varphi(t) & =\frac{1}{\Gamma(n-1-r)} \int_{t}^{T}(s-t)^{n-2-r} T^{\lambda}(T-s)^{\lambda} d s \\
& =\frac{T^{-\lambda}}{\Gamma(n-1-r)} \int_{t}^{T}[(T-t)-(T-s)]^{n-2-r}(T-s)^{\lambda} d s \\
& =\frac{T^{-\lambda}}{\Gamma(n-1-r)}(T-t)^{n-2-r} \int_{t}^{T}\left(1-\frac{T-s}{T-t}\right)^{n-2-r}(T-s)^{\lambda} d s .
\end{aligned}
$$

If we consider the change of variable $\tau=\frac{T-s}{T-t}$, then we get

$$
\begin{aligned}
I_{T}^{n-1-r} \varphi(t) & =\frac{T^{-\lambda}}{\Gamma(n-1-r)}(T-t)^{n-1-r+\lambda} \int_{0}^{1}(1-\tau)^{n-2-r} \tau^{\lambda} d \tau \\
& =\frac{T^{-\lambda}}{\Gamma(n-1-r)}(T-t)^{n-1-r+\lambda} B(n-1-r, \lambda+1),
\end{aligned}
$$

where $B(\cdot, \cdot)$ is the Beta function (see, for example [3], p. 26). Taking into account the property

$$
B(a, b)=\frac{\Gamma(a) \Gamma(b)}{\Gamma(a+b)}, \quad a, b>0,
$$

we obtain

$$
\begin{aligned}
I_{T}^{n-1-r} \varphi(t) & =\frac{T^{-\lambda} \Gamma(n-1-r) \Gamma(\lambda+1)}{\Gamma(n-1-r) \Gamma(n-r+\lambda)}(T-t)^{n-1-r+\lambda} \\
& =\frac{\Gamma(\lambda+1)}{\Gamma(n-r+\lambda)} T^{-\lambda}(T-t)^{n-1-r+\lambda},
\end{aligned}
$$

which proves (8). Acting a differentiation, we achieve the mathematical form

$$
\left(I_{T}^{n-1-r} \varphi\right)^{\prime}(t)=-\frac{(n-1-r+\lambda) \Gamma(\lambda+1)}{\Gamma(n-r+\lambda)} T^{-\lambda}(T-t)^{n-2-r+\lambda} .
$$

Moreover, taking into account the property

$$
a \Gamma(a)=\Gamma(a+1), \quad a>0,
$$

we obtain

$$
\left(I_{T}^{n-1-r} \varphi\right)^{\prime}(t)=-\frac{\Gamma(\lambda+1)}{\Gamma(n-r-1+\lambda)} T^{-\lambda}(T-t)^{n-2-r+\lambda} .
$$

Consequently, (9) holds true for $i=1$. A induction strategy ensures us that (9) is true for all $i=1,2, \ldots, n$. It remains to use (9) together with a similar calculation as that for the proof of (8), and, hence, (10) can be established.

\section{Proofs of the Main Results}

\subsection{The Case $\vartheta \equiv 0$}

Our strategy here is to distinguish two cases. Therefore, we first provide the proof in the case where $y_{j}>0$ for some $j \in\{1,2, \ldots, n-1\}$.

Proof of Theorem 1. The proof is developed by contradiction. We fix a global solution to (1), namely $y \in A C^{n}\left(\mathbb{R}_{+}\right)$. For a finite positive value $0<T<\infty$, we consider a integral where the integrand is a simple product of the fractional differential inequality in (1) by the function $\varphi$. Recall that $\varphi$ is the test function given by (7), and, hence, we have

$$
\int_{0}^{T}\left|{ }^{C} D_{0}^{\gamma} y(t)\right|^{q} \varphi(t) d t \leq \int_{0}^{T}{ }^{C} D_{0}^{1+\alpha} y(t) \varphi(t) d t+\int_{0}^{T}{ }^{C} D_{0}^{1+\beta} y(t) \varphi(t) d t .
$$


It is not restrictive to assume the following condition

$$
n-2<\alpha \leq \beta<n-1 \text {. }
$$

The precise information in Lemma 2 can be used to get

$$
\begin{aligned}
\int_{0}^{T}{ }^{C} D_{0}^{1+\alpha} y(t) \varphi(t) d t & =\int_{0}^{T} I_{0}^{n-1-\alpha} y^{(n)}(t) \varphi(t) d t \\
& =\int_{0}^{T} y^{(n)}(t) I_{T}^{n-1-\alpha} \varphi(t) d t
\end{aligned}
$$

Integrating by parts $((n-1)$-times) and using the information in Lemma 3 , namely Equations (8) and (9), we deduce that

$$
\begin{aligned}
\int_{0}^{T} C^{C} D_{0}^{1+\alpha} y(t) \varphi(t) d t= & \sum_{k=1}^{n-1}(-1)^{k} y_{n-k}\left(I_{T}^{n-1-\alpha} \varphi\right)^{(k-1)}(0) \\
& +(-1)^{n-1} \int_{0}^{T} y^{\prime}(t)\left(I_{T}^{n-1-\alpha} \varphi\right)^{(n-1)}(t) d t .
\end{aligned}
$$

In view of the preliminaries in Section 2, we adopted the notation $f^{(0)}=f$. Next, by Lemma 1, from

$$
y^{\prime}(t)=\left(y(t)-y_{0}\right)^{\prime}=\left(\int_{0}^{t} y^{\prime}(s) d s\right)^{\prime}=\left(I_{0}^{1} y^{\prime}\right)(t)=\left[I_{0}^{\gamma}\left(I_{0}^{1-\gamma} y^{\prime}\right)\right]^{\prime}(t)
$$

considering a integration by parts, jointly with the information in Lemma 2, we obtain

$$
\begin{aligned}
& \int_{0}^{T} y^{\prime}(t)\left(I_{T}^{n-1-\alpha} \varphi\right)^{(n-1)}(t) d t=\int_{0}^{T}\left[I_{0}^{\gamma}\left(I_{0}^{1-\gamma} y^{\prime}\right)\right]^{\prime}(t)\left(I_{T}^{n-1-\alpha} \varphi\right)^{(n-1)}(t) d t \\
& =\left[\left(y(t)-y_{0}\right)\left(I_{T}^{n-1-\alpha} \varphi\right)^{(n-1)}(t)\right]_{t=0}^{T} \\
& -\int_{0}^{T} I_{0}^{\gamma}\left(I_{0}^{1-\gamma} y^{\prime}\right)(t)\left(I_{T}^{n-1-\alpha} \varphi\right)^{(n)}(t) d t \\
& =-\int_{0}^{T}{ }^{C} D_{0}^{\gamma} y(t) I_{T}^{\gamma}\left[\left(I_{T}^{n-1-\alpha} \varphi\right)^{(n)}\right](t) d t \text {. }
\end{aligned}
$$

The Equation (13) leads to

$$
\begin{aligned}
\int_{0}^{T}{ }^{C} D_{0}^{1+\alpha} y(t) \varphi(t) d t= & \sum_{k=1}^{n-1}(-1)^{k} y_{n-k}\left(I_{T}^{n-1-\alpha} \varphi\right)^{(k-1)}(0) \\
& +(-1)^{n} \int_{0}^{T} C^{C} D_{0}^{\gamma} y(t) I_{T}^{\gamma}\left[\left(I_{T}^{n-1-\alpha} \varphi\right)^{(n)}\right](t) d t .
\end{aligned}
$$

In a similar fashion, we deduce that

$$
\begin{aligned}
\int_{0}^{T} C^{C} D_{0}^{1+\beta} y(t) \varphi(t) d t= & \sum_{k=1}^{n-1}(-1)^{k} y_{n-k}\left(I_{T}^{n-1-\beta} \varphi\right)^{(k-1)}(0) \\
& +(-1)^{n} \int_{0}^{T} C^{C} D_{0}^{\gamma} y(t) I_{T}^{\gamma}\left[\left(I_{T}^{n-1-\beta} \varphi\right)^{(n)}\right](t) d t
\end{aligned}
$$

Now (11), together with (14) and (15), gives us

$$
\begin{aligned}
& \int_{0}^{T}\left|{ }^{C} D_{0}^{\gamma} y(t)\right|^{q} \varphi(t) d t+\sum_{k=1}^{n-1}(-1)^{k+1} y_{n-k}\left[\left(I_{T}^{n-1-\alpha} \varphi\right)^{(k-1)}(0)+\left(I_{T}^{n-1-\beta} \varphi\right)^{(k-1)}(0)\right] \\
& \leq \int_{0}^{T}\left|{ }^{C} D_{0}^{\gamma} y(t)\right|\left|I_{T}^{\gamma}\left[\left(I_{T}^{n-1-\alpha} \varphi\right)^{(n)}\right](t)\right| d t+\int_{0}^{T}\left|{ }^{C} D_{0}^{\gamma} y(t)\right|\left|I_{T}^{\gamma}\left[\left(I_{T}^{n-1-\beta} \varphi\right)^{(n)}\right](t)\right| d t .
\end{aligned}
$$


The $\varepsilon$-Young inequality with $\varepsilon=\frac{1}{2}$ (of course any different $\varepsilon \in(0,1)$ is suitable) implies that

$$
\begin{aligned}
& \int_{0}^{T}\left|{ }^{C} D_{0}^{\gamma} y(t)\right|\left|I_{T}^{\gamma}\left[\left(I_{T}^{n-1-\alpha} \varphi\right)^{(n)}\right](t)\right| d t \\
& \leq \frac{1}{2} \int_{0}^{T}\left|{ }^{C} D_{0}^{\gamma} y(t)\right|^{q} \varphi(t) d t+C \int_{0}^{T} \varphi(t)^{\frac{-1}{q-1}}\left|I_{T}^{\gamma}\left[\left(I_{T}^{n-1-\alpha} \varphi\right)^{(n)}\right](t)\right|^{\frac{q}{q-1}} d t .
\end{aligned}
$$

We underline that the constant $C>0$ can change from line to line. A similar argument, as the one developed above, gives us the inequality

$$
\begin{aligned}
& \int_{0}^{T}\left|{ }^{C} D_{0}^{\gamma} y(t)\right|\left|I_{T}^{\gamma}\left[\left(I_{T}^{n-1-\beta} \varphi\right)^{(n)}\right](t)\right| d t \\
& \leq \frac{1}{2} \int_{0}^{T}\left|{ }^{C} D_{0}^{\gamma} y(t)\right|^{q} \varphi(t) d t+C \int_{0}^{T} \varphi(t)^{\frac{-1}{q-1}}\left|I_{T}^{\gamma}\left[\left(I_{T}^{n-1-\beta} \varphi\right)^{(n)}\right](t)\right|^{\frac{q}{q-1}} d t .
\end{aligned}
$$

Combining (16)-(18), we deduce that

$$
\sum_{k=1}^{n-1}(-1)^{k+1} y_{n-k}\left[\left(I_{T}^{n-1-\alpha} \varphi\right)^{(k-1)}(0)+\left(I_{T}^{n-1-\beta} \varphi\right)^{(k-1)}(0)\right] \leq C\left(I_{1}(\varphi)+I_{2}(\varphi)\right),
$$

where

$$
I_{1}(\varphi)=\int_{0}^{T} \varphi(t)^{\frac{-1}{q-1}}\left|I_{T}^{\gamma}\left[\left(I_{T}^{n-1-\alpha} \varphi\right)^{(n)}\right](t)\right|^{\frac{q}{q-1}} d t
$$

and

$$
I_{2}(\varphi)=\int_{0}^{T} \varphi(t)^{\frac{-1}{q-1}}\left|I_{T}^{\gamma}\left[\left(I_{T}^{n-1-\beta} \varphi\right)^{(n)}\right](t)\right|^{\frac{q}{q-1}} d t
$$

Next, by (9), we have

$$
\left(I_{T}^{n-1-\alpha} \varphi\right)^{(k-1)}(0)=(-1)^{k-1} \frac{\Gamma(\lambda+1)}{\Gamma(n-\alpha+\lambda-k+1)} T^{n-\alpha-k}, \quad k=1,2, \ldots, n-1 .
$$

Confirming a similar reasoning as above, we get

$$
\left(I_{T}^{n-1-\beta} \varphi\right)^{(k-1)}(0)=(-1)^{k-1} \frac{\Gamma(\lambda+1)}{\Gamma(n-\beta+\lambda-k+1)} T^{n-\beta-k}, \quad k=1,2, \ldots, n-1 .
$$

It follows that

$$
\begin{aligned}
& \sum_{k=1}^{n-1}(-1)^{k+1} y_{n-k}\left[\left(I_{T}^{n-1-\alpha} \varphi\right)^{(k-1)}(0)+\left(I_{T}^{n-1-\beta} \varphi\right)^{(k-1)}(0)\right] \\
& =\sum_{k=1}^{n-1} y_{n-k}\left[\frac{\Gamma(\lambda+1)}{\Gamma(n-\alpha+\lambda-k+1)} T^{n-\alpha-k}+\frac{\Gamma(\lambda+1)}{\Gamma(n-\beta+\lambda-k+1)} T^{n-\beta-k}\right] \\
& =\sum_{i=1}^{n-1} y_{i}\left[\frac{\Gamma(\lambda+1)}{\Gamma(\lambda-\alpha+i+1)} T^{i-\alpha}+\frac{\Gamma(\lambda+1)}{\Gamma(\lambda-\beta+i+1)} T^{i-\beta}\right] \\
& \geq \frac{\Gamma(\lambda+1)}{\Gamma(\lambda-\alpha+j+1)} y_{j} T^{j-\alpha} .
\end{aligned}
$$

As the next step, we aim to calculate the terms $I_{i}(\varphi), i=1,2$. Adopting, again, the test function defined in (7) and working in the setting of Lemma 3 , for $t \in(0, T)$ we deduce that

$$
\varphi(t)^{\frac{-1}{q-1}}\left|I_{T}^{\gamma}\left[\left(I_{T}^{n-1-\alpha} \varphi\right)^{(n)}\right](t)\right|^{\frac{q}{q-1}}=\left[\frac{\Gamma(\lambda+1)}{\Gamma(\gamma+\lambda-\alpha)}\right]^{\frac{q}{q-1}} T^{-\lambda}(T-t)^{\lambda+\frac{(\gamma-\alpha-1) q}{q-1}} .
$$


Integrating over $(0, T)$, we get

$$
I_{1}(\varphi)=\left[\frac{\Gamma(\lambda+1)}{\Gamma(\gamma+\lambda-\alpha)}\right]^{\frac{q}{q-1}} T^{\frac{(\gamma-\alpha-1) q}{q-1}} \int_{0}^{T}\left(1-\frac{t}{T}\right)^{\lambda+\frac{(\gamma-\alpha-1) q}{q-1}} d t .
$$

We choose the change of variable $s=\frac{t}{T}$ to deduce that

$$
I_{1}(\varphi)=\left[\frac{\Gamma(\lambda+1)}{\Gamma(\gamma+\lambda-\alpha)}\right]^{\frac{q}{q-1}} T^{\frac{(\gamma-\alpha-1) q}{q-1}+1} \int_{0}^{s}(1-s)^{\lambda+\frac{(\gamma-\alpha-1) q}{q-1}} d s
$$

that is,

$$
I_{1}(\varphi)=C T^{\frac{(\gamma-\alpha-1) q}{q-1}+1} .
$$

Moreover, in a similar fashion we get

$$
I_{2}(\varphi)=C T^{\frac{(\gamma-\beta-1) q}{q-1}+1} .
$$

Working on the sum of (23) and (24), we obtain the inequality

$$
I_{1}(\varphi)+I_{2}(\varphi) \leq C T^{\frac{(\gamma-\alpha-1) q}{q-1}+1}\left(1+T^{\frac{-(\beta-\alpha) q}{q-1}}\right) .
$$

Taking into account the limitation given by (12), we conclude that for sufficiently large value of $T$,

$$
I_{1}(\varphi)+I_{2}(\varphi) \leq C T^{\frac{(\gamma-\alpha-1) q}{q-1}+1} .
$$

The competing effects of (19), (22), and (25) lead to

$$
y_{j} \leq C T^{\frac{(\gamma-\alpha-1) q}{q-1}+1-j+\alpha}
$$

We observe that

$$
\frac{(\gamma-\alpha-1) q}{q-1}+1-j+\alpha<0, \quad \text { for all } q>1 .
$$

Hence, passing to the limit as $T$ goes to $\infty$ in (26), we achieve

$$
y_{j} \leq 0
$$

but this conclusion contradicts the hypothesis on the positivity of $y_{j}$. Hence we conclude that Theorem 1 has to be true.

The second main result of our interest deals with the case of $y_{i}=0$, for all $i \in$ $\{1,2, \ldots, n-1\}$. Now, it is interesting to see how the above proof changes and thus arrives at a different theorem.

Proof of Theorem 2. We bring to the attention of the reader that in this case, $y \equiv y_{0}$ is a global solution to problem (1).

Now, let $y \in A C^{n}\left(\mathbb{R}_{+}\right)$be a global solution to (1). For a finite positive value $0<T<\infty$, we use again the strategy to consider a integral where the integrand is the product of the fractional differential inequality in (1) by the test function $\varphi$ in (7). We integrate over the interval $(0, T)$, to retrieve the inequality (16). Since $y_{i}=0, i=1,2, \ldots, n-1$, we deduce that

$$
\begin{aligned}
& \int_{0}^{T}\left|{ }^{C} D_{0}^{\gamma} y(t)\right|^{q} \varphi(t) d t \\
& \leq \int_{0}^{T}\left|{ }^{C} D_{0}^{\gamma} y(t)\right|\left|I_{T}^{\gamma}\left[\left(I_{T}^{n-1-\alpha} \varphi\right)^{(n)}\right](t)\right| d t+\int_{0}^{T}\left|{ }^{C} D_{0}^{\gamma} y(t)\right|\left|I_{T}^{\gamma}\left[\left(I_{T}^{n-1-\beta} \varphi\right)^{(n)}\right](t)\right| d t .
\end{aligned}
$$


Applying the $\varepsilon$-Young inequality with $0<\varepsilon<\frac{1}{2}$, and proceeding step by step as in the proof of Theorem 1, we arrive to the inequality

$$
\int_{0}^{T}\left|{ }^{C} D_{0}^{\gamma} y(t)\right|^{q} \varphi(t) d t \leq C\left(I_{1}(\varphi)+I_{2}(\varphi)\right),
$$

where $I_{i}(\varphi), i=1,2$, are given by (20) and (21). The test function in (7) and the inequality in (25), give us

$$
\int_{0}^{T}\left|{ }^{C} D_{0}^{\gamma} y(t)\right|^{q}\left(1-\frac{t}{T}\right)^{\lambda} d t \leq C T^{\frac{(\gamma-\alpha-1) q}{q-1}+1},
$$

for sufficiently large $T$. This time we observe that

$$
\frac{(\gamma-\alpha-1) q}{q-1}+1<0 \Longleftrightarrow q(\gamma-\alpha)<1
$$

Using Fatou's lemma, the infimum limit, for $T$ going to infinite, whenever $\gamma \leq \alpha$ in (27), leads to

$$
\begin{aligned}
& \int_{0}^{\infty}\left|{ }^{C} D_{0}^{\gamma} y(t)\right|^{q} d t=0, \\
\Rightarrow & { }^{C} D_{0}^{\gamma} y(t)=0,
\end{aligned}
$$

for almost everywhere $t \geq 0$. The information in Lemma 1 implies that

$$
0=I_{0}^{\gamma C} D_{0}^{\gamma} y(t)=I_{0}^{\gamma}\left(I_{0}^{1-\gamma} y^{\prime}\right)(t)=I_{0}^{1} y^{\prime}(t)=y(t)-y_{0},
$$

for almost everywhere $t \geq 0$. Since $y$ is continuous (recall that $y \in A C^{n}\left(\mathbb{R}_{+}\right)$), we deduce that $y(t)=y_{0}$, for all $t \geq 0$. Therefore, we conclude that $y \equiv y_{0}$ is the only global solution to problem (1). This fact establishes the first result of Theorem 2 (namely part (i)).

It remains to show that also the part (ii) of Theorem 2 holds true. Thus, we focus on the case where $\gamma>\alpha$. By the double implication in (28) we get

$$
\frac{(\gamma-\alpha-1) q}{q-1}+1<0 \Longleftrightarrow q<\frac{1}{\gamma-\alpha} .
$$

If $1<q<\frac{1}{\gamma-\alpha}$, repeating the same argument as above, we obtain $y(t)=y_{0}$, for all $t \geq 0$, which proves that $y \equiv y_{0}$ is the only global solution to problem (1). This concludes the proof of part (ii) of Theorem 2.

\subsection{The Case $\vartheta \not \equiv 0$}

Proof of Theorem 3. Let $y \in A C^{n}\left(\mathbb{R}_{+}\right)$be a global solution to (1). Remark that $\vartheta \geq 0$ leads to

$$
{ }^{C} D_{0}^{1+\alpha} y(t)+{ }^{C} D_{0}^{1+\beta} y(t) \geq\left|{ }^{C} D_{0}^{\gamma} y(t)\right|^{q},
$$

for almost everywhere $t \geq 0$. Hence, part (i) of Theorem 3 follows from part (i) of Theorem 2 .

Now, consider the case $\gamma>\alpha$. Following the proof of part (ii) of Theorem 2, we arrive to the inequality

$$
\int_{0}^{T} \vartheta(t) \varphi(t) d t+\int_{0}^{T}\left|{ }^{C} D_{0}^{\gamma} y(t)\right|^{q} \varphi(t) d t \leq C\left(I_{1}(\varphi)+I_{2}(\varphi)\right),
$$

which yields

$$
\int_{0}^{T} \vartheta(t) \varphi(t) d t \leq C\left(I_{1}(\varphi)+I_{2}(\varphi)\right)
$$


On the other hand, since $\vartheta \geq 0$,

$$
\begin{aligned}
\int_{0}^{T} \vartheta(t) \varphi(t) d t & =\int_{0}^{T} \vartheta(t)\left(1-\frac{t}{T}\right)^{\lambda} d t \\
& \geq \int_{0}^{\frac{T}{2}} \vartheta(t)\left(1-\frac{t}{T}\right)^{\lambda} d t \\
& \geq 2^{-\lambda} \int_{0}^{\frac{T}{2}} \vartheta(t) d t .
\end{aligned}
$$

Hence, (29) leads to

$$
\int_{0}^{\frac{T}{2}} \vartheta(t) d t \leq C\left(I_{1}(\varphi)+I_{2}(\varphi)\right)
$$

Next, The test function in (7) and the inequality in (25), give us

$$
T^{-\frac{(\gamma-\alpha-1) q}{q-1}-1} \int_{0}^{\frac{T}{2}} \vartheta(t) d t \leq C,
$$

which contradicts (4). This concludes the proof of part (ii) of Theorem 3.

Finally, we consider the class of functions $\vartheta$ given by (5).

Proof of Corollary 1. It is not difficult to show that

$$
T^{-\frac{(\gamma-\alpha-1) q}{q-1}-1} \int_{0}^{T} \vartheta(t) d t \sim T^{\rho-\frac{(\gamma-\alpha-1) q}{q-1}}, \quad \text { as } T \rightarrow \infty .
$$

Moreover, from $\gamma>\alpha$ and $\gamma-\alpha-1<\rho \leq 0$, we obtain

$$
\rho-\frac{(\gamma-\alpha-1) q}{q-1}>0, \quad q>1 .
$$

Hence, (4) is satisfied, and the desired result follows from part (ii) of Theorem 3.

\section{Conclusions}

Mathematical methods can be helpful to identify the characteristics and origins of materials and flow phenomena across various applications. Understanding the mechanisms underlying the effects of physical structures, is crucial for experimentalists interested to identify the properties of particular systems. Sufficient criteria of nonexistence of global solutions to higher order fractional differential inequalities with certain nonlinearities have been presented. Here, using nonlinear capacity method, a specific test function is adopted and some a priori estimates of solutions are derived. The qualitative behavior of global solutions of the inequality is investigated on the space of absolutely continuous functions over the positive semiaxis $\mathbb{R}_{+}$. In particular, a nonlinearity involving Caputo fractional derivative has been considered. The obtained abstract results are useful to attaining analytical models with functions which have continuous derivatives up to order $n-1$ on $\mathbb{R}_{+}(n \geq 2)$. Further investigations will be devoted to discuss the impact of different nonlinearities on the non-solvability of the inequality. Inequalities as (1) could be also used to develop certain special means which have a role in confirming the accuracy of abstract results (for more details see [22]). Finally, the use of a parametric nonlinearity could lead to bifurcation-type results, where the effects on the (non-)solvability behavior depend on the parameter, that moves on the real line.

Author Contributions: Investigation, M.J., B.S. and C.V. All authors have read and agreed to the published version of the manuscript.

Funding: The second author is supported by Researchers Supporting Project number (RSP-2021/4), King Saud University, Riyadh, Saudi Arabia. 
Conflicts of Interest: The authors declare no conflicts of interest.

\section{References}

1. Samko, S.G.; Kilbas, A.A.; Marichev, O.I. Fractional Integrals and Derivatives: Theory and Applications; Gordon and Breach: Yverdon, Switzerland, 1993.

2. Zhou, Y. Basic Theory of Fractional Differential Equations; World Scientific: Singapore, 2014.

3. Kilbas, A.A.; Srivastava, H.M.; Trujillo, J.J. Theory and Applications of Fractional Differential Equations; Elsevier: Amsterdam, The Netherlands, 2006.

4. Almuthaybiri, S.S.; Tisdell, C.C. Global existence theory for fractional differential equations: New advances via continuation methods for contractive maps. Analysis 2019, 39, 117-128. [CrossRef]

5. Cong, N.D.; Tuan, H.T. Existence, uniqueness, and exponential boundedness of global solutions to delay fractional differential equations. Mediterr. J. Math. 2007, 14, 1-12. [CrossRef]

6. Zhang, P.; Hao, X.; Liu, L. Existence and uniqueness of the global solution for a class of nonlinear fractional integro-differential equations in a Banach space. Adv. Differ. Equ. 2019, 135, 1-10. [CrossRef]

7. Zhu, T. Fractional integral inequalities and global solutions of fractional differential equations. Electron. J. Qual. Theory. Differ. Equ. 2020, 5, 1-16. [CrossRef]

8. Alsaedi, A.; Ahmad, B.; Kirane, M.; Al Musalhi, F.S.K.; Alzahrani, F. Blowing-up solutions for a nonlinear time-fractional system. Bull. Math. Sci. 2017, 7, 201-210. [CrossRef]

9. Furati, K.M.; Kirane, M. Necessary conditions for the existence of global solutions to systems of fractional differential equations. Fract. Calc. Appl. Anal. 2008, 11, 281-298.

10. Jleli, M.; Samet, B. Nonexistence results for some classes of nonlinear fractional differential inequalities. J. Funct. Spaces 2020, 22, 1-8. [CrossRef]

11. Kirane, M.; Medved, M.; Tatar, N.-E. On the nonexistence of blowing-up solutions to a fractional functional differential equations. Georgian Math. J. 2012, 19, 127-144. [CrossRef]

12. Mennouni, A.; Youkana, A. Finite time blow-up of solutions for a nonlinear system of fractional differential equations. Electron. J. Differ. Equ. 2017, 152, 1-15.

13. Samet, B. Nonexistence of global solutions for a class of sequential fractional differential inequalities. Eur. Phys. J. Spec. Top. 2017, 226, 3513-3524. [CrossRef]

14. Zhao, X.; Chai, C.; Ge, W. Existence and nonexistence results for a class of fractional boundary value problems. J. Appl. Math. Comput. 2013, 41, 17-31. [CrossRef]

15. Heydari, M.H.; Atangana, A.; Avazzadeh, Z. Numerical solution of nonlinear fractal-fractional optimal control problems by Legendre polynomials. Math. Methods Appl. Sci. 2021, 44, 2952-2963. [CrossRef]

16. Liu, C.; Zhang, X.; Wu, B. Numerical solution of fractional differential equations by semiorthogonal B-splines wavelets. Math. Methods Appl. Sci. 2021, 44, 2697-2710. [CrossRef]

17. Mitidieri, E.; Pohozaev, S.I. A priori estimates and blow-up of solutions to nonlinear partial differential equations and inequalities. Proc. Steklov Inst. Math. 2001, 234, 1-383.

18. Sumelka, W.; Luczak, B.; Gajewski, T.; Voyiadjis, G.Z. Modelling of AAA in the framework of time-fractional damage hyperelasticity. Int. J. Solids Struct. 2020, 206, 30-42. [CrossRef]

19. Zhou, Y.; Zhang, Y. Noether symmetries for fractional generalized Birkhoffian systems in terms of classical and combined Caputo derivatives. Acta Mech. 2020, 231, 3017-3029. [CrossRef]

20. Podlubny, I. Fractional Differential Equations; Mathematics in Science and Engineering; Academic Press: Cambridge, MA, USA, 1999; Volume 198.

21. Qureshi, S. Real life application of Caputo fractional derivative for measles epidemiological autonomous dynamical system. Chaos Solitons Fractals 2020, 134, 109744. [CrossRef]

22. Chen, L.; Zhang, J.; Saleem, M.S.; Ahmed, I.; Waheed, S.; Pan, L. Fractional integral inequalities for $h$-convex functions via Caputo-Fabrizio operator. AIMS Math. 2021, 6, 6377-6389. [CrossRef] 